\title{
Conceptual Representations of Action in the Lateral Temporal Cortex
}

\author{
Joseph W. Kable, Irene P. Kan, Ashley Wilson, \\ Sharon L. Thompson-Schill, and Anjan Chatterjee
}

\begin{abstract}
Retrieval of conceptual information from action pictures causes greater activation than from object pictures bilaterally in human motion areas (MT/MST) and nearby temporal regions. By contrast, retrieval of conceptual information from action words causes greater activation in left middle and superior temporal gyri, anterior and dorsal to the MT/MST. We performed two fMRI experiments to replicate and extend these findings regarding action words. In the first experiment, subjects performed conceptual judgments of action and object words under conditions that stressed visual semantic information. Under these conditions, action words again activated posterior temporal regions close to, but not identical with, the $\mathrm{MT} / \mathrm{MST}$. In the second experiment, we included conceptual
\end{abstract}

\section{INTRODUCTION}

A large body of work within cognitive neuroscience has examined how the brain mediates conceptual knowledge of the world. Starting from the observation that knowledge of specific categories of objects can be selectively impaired by brain damage, a range of competing theories have been proposed for the neural mediation of conceptual knowledge. One class of theories argues that category-specific deficits do not result from category-specific organization per se, but rather because different kinds of information are more relevant to concepts in different conceptual categories (Gainotti, 2000; Saffran \& Schwartz, 1994; Warrington \& Shallice, 1984). According to this view, conceptual knowledge is mediated by distributed neural networks, partially dissociable according to the kind of information represented, such as particular sensory or motor attributes. Variations within this general class of theories have been elaborated to explain how such conceptual representations interact with perceptual or language systems to allow for category-specific deficits restricted to language or perception (Damasio, Tranel, Grabowski, Adolphs, \& Damasio, 2004; Simmons \& Barsalou, 2003;

University of Pennsylvania judgments of manipulable object words in addition to judgments of action and animal words. Both action and manipulable object judgments caused greater activity than animal judgments in the posterior middle temporal gyrus. Both of these experiments support the hypothesis that middle temporal gyrus activation is related to accessing conceptual information about motion attributes, rather than alternative accounts on the basis of lexical or grammatical factors. Furthermore, these experiments provide additional support for the notion of a concrete to abstract gradient of motion representations with the lateral occipito-temporal cortex, extending anterior and dorsal from the MT/MST towards the peri-sylvian cortex.

Pulvermuller, 1999). A second class of theories proposes that category-specific deficits are evidence of categoryspecific organization, and that the brain contains domain-specific knowledge systems shaped by evolution and specialized for particular categories of knowledge (Caramazza \& Mahon, 2003). Finally, a third class of theories also argues that conceptual knowledge is mediated by distributed neural networks, but these networks are not organized along sensorimotor lines (Tyler \& Moss, 2001; Caramazza, Hillis, Rapp, \& Romani, 1990). Rather, category-specific deficits arise because of the structure of knowledge in different conceptual categories (intercorrelations vs. uniqueness of semantic features), and how such structure breaks down with damage to a unitary conceptual system.

However, most of the tests pitting these competing theories have focused on knowledge of different categories of objects. Comparatively less attention has been given to what might be considered a broader distinction between actions and objects. In language, verbs commonly represent actions, and nouns commonly represent objects. Brain damage can independently impair conceptual knowledge of actions and objects (Daniele, Giustolisi, Silveri, Colosimo, \& Gainotti, 1994; Miceli, Silveri, Nocentini, \& Caramazza, 1988; McCarthy \& Warrington, 1985), and a few functional imaging studies have found distinct brain areas that are engaged to a 
greater degree by actions or objects (Tranel, Martin, Damasio, Grabowski, \& Hichwa, in press; Kable, LeaseSpellmeyer, \& Chatterjee, 2002; Damasio, Grabowski, Tranel, Ponto, et al., 2001; Perani, Cappa, et al., 1999; Martin, Haxby, Lalonde, Wiggs, \& Ungerleider, 1995). An understanding of the neural substrates of actions, compared to different categories of objects, should provide important evidence for evaluating theories of conceptual knowledge systems, as well as a more complete picture of the neural mediation of conceptual knowledge.

We directly investigated the neural substrates of action knowledge in a previous fMRI study (Kable, LeaseSpellmeyer, et al., 2002). Subjects saw three pictures or three words simultaneously, one at the top of the screen and two at the bottom, and they were asked to determine which of the two concepts presented at the bottom was most similar to the one presented at the top. For example, subjects would decide that "digging" was more similar to "shoveling" than "listening" or that "doctor" was more similar to "cop" than "zucchini." Subjects performed the same task once with pictures and once with words. When subjects performed the task with pictures, there was greater activity for actions, compared to objects, bilaterally in human motion area MT/MST and in nearby areas of the middle temporal gyrus. When subjects performed the task with words, there was greater activity for actions, compared to objects, in the left middle and superior temporal gyri, anterior and dorsal to the activation for pictures. Action words, unlike action pictures, did not preferentially activate area MT/MST.

These results suggest that parts of the lateral occipitotemporal cortex are particularly important for mediating conceptual knowledge of actions. In addition, considering the involvement of the MT/MST, the sensorimotor theory of conceptual knowledge provides the most natural explanation for these findings. Within this framework, we argued that the lateral occipito-temporal cortex likely mediates different representations of motion, which are presumably more relevant for action concepts compared to object concepts. Furthermore, we suggested that these motion representations are organized on a concrete to abstract gradient, with more concrete representations preferentially accessed from pictures located more posteriorly near the MT/MST, and more abstract representations preferentially accessed from words located more anteriorly near the peri-sylvian cortex.

However, there are alternative possible explanations for our findings concerning action words, especially given the small overlap between areas showing greater activation for action words and action pictures. Action words may have failed to activate area MT/MST because the judgments for the particular stimuli used did not stress detailed motion information, and not because of any intrinsic material specificity. Furthermore, activation differences in the lateral temporal cortex may have been due to lexical differences between the action and object words, rather than the differences in conceptual category. In particular, all of the action words were verbs, whereas all of the object words were nouns, so perhaps grammatical processing accounts for these activation differences. Although other investigators have failed to detect significant differences between verbs and nouns in the lateral temporal cortex (Tyler, Bright, Fletcher, \& Stamatakis, 2004; Tyler, Stamatakis, et al., 2003; Tyler, Russell, Fadili, \& Moss, 2001), this failure highlights the additional possibility that our previous findings with action words may have been idiosyncratic to the particular stimuli or subjects.

We designed two fMRI experiments to test these alternative hypotheses. Both experiments test the hypothesis that activity for words in the lateral temporal cortex is driven by semantic differences as we previously argued. In the first experiment, we designed new word triads for the conceptual judgment task that focused subjects' attention on a more constrained set of visual attributes (see Figure 1). In our previous study, because the distractor item on each trial was often distantly related to the other two items ("listening" compared to "digging" and "shoveling"), subjects could have made their judgments with only minimal access to visual attributes of different actions (unlike "listening," "digging" and "shoveling" involve motion). We designed new stimuli in which all three words were closely related, to increase the importance of visual attributes in making the judgment. For example, subjects would judge that "skipping" is more similar to "bouncing" than "rolling," as both skipping and bouncing have similar manners of motion, or they would judge that "zebra" is more similar to "horse" than "cow," as both zebras and horses have similar forms. If activation in the lateral temporal cortex is associated with accessing semantic information about actions, then we would expect to replicate our original finding of greater activation for action words compared to object words. Furthermore, if our hypothesis about a gradient of action representations is correct, we would expect the activation for action words to be closer to the MT/MST in the current experiment, because these stimuli emphasized visual information about actions at a greater level of detail than in our previous study.

In the second experiment, we again used the conceptual judgment task, but with three different categories of words: actions, animals, and manipulable objects. Although the words for both animals and manipulable objects are nouns, manipulable object concepts, more than animal concepts, rely on information about movement (Damasio, Tranel, et al., 2004; Saffran, Coslett, \& Keener, 2003; Buxbaum \& Saffran, 2002; McRae, de Sa, \& Seidenberg, 1997). Unlike animals, most manipulable objects are strongly associated with a constrained set of actions. So, if the previously observed activation differences between action and object words were driven 
Figure 1. Schematic of the experimental paradigm. Subjects were presented with 18 -sec blocks of conceptual judgments, interspersed with baseline blocks.

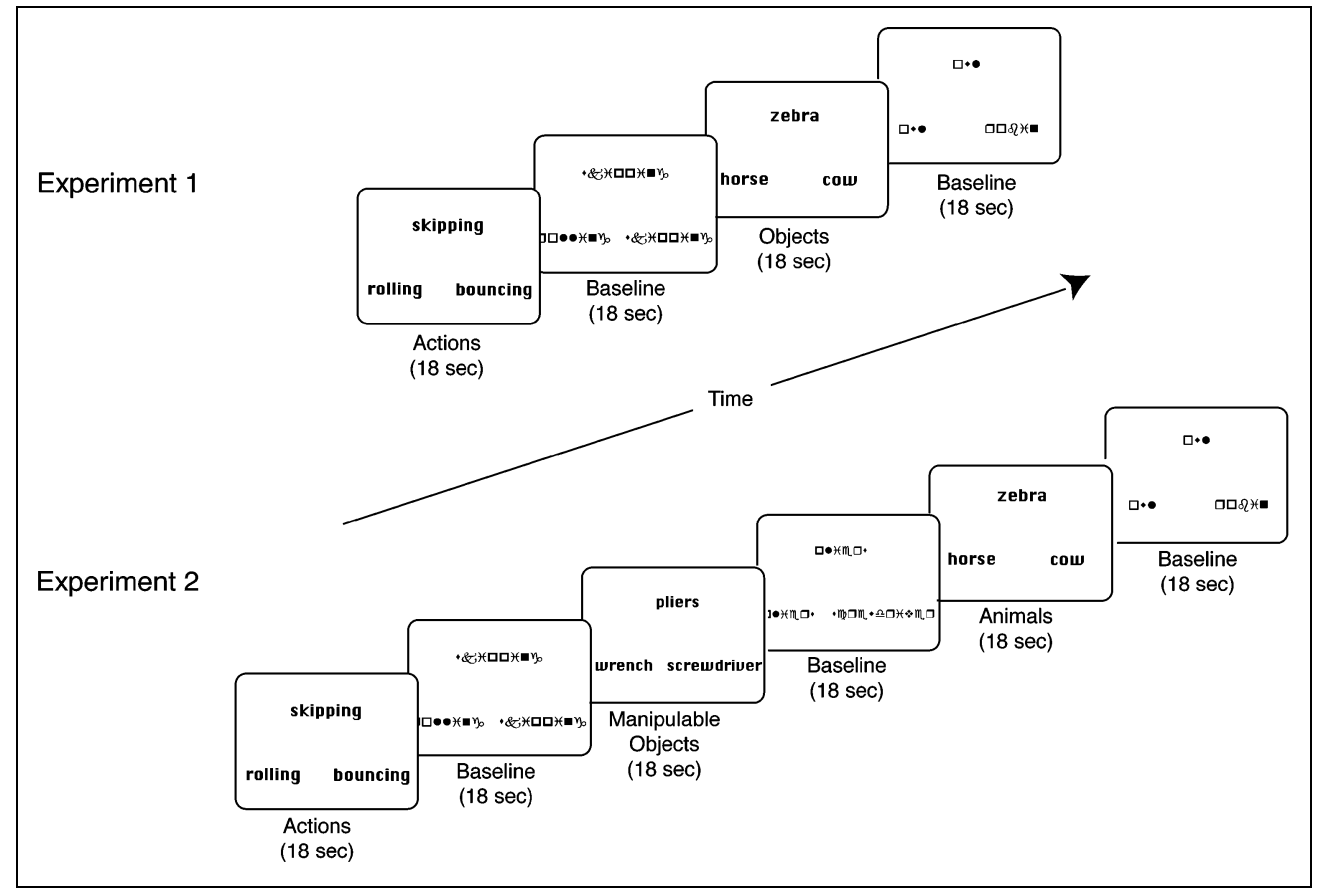

by visual attributes associated with concepts, then both action and manipulable object words should cause greater activation than animals in the lateral temporal cortex. However, if these activation differences were driven by linguistic differences evoked by grammatical class, then action words should cause greater activity than both animal and manipulable object words in the lateral temporal cortex.

\section{RESULTS}

\section{Experiment 1}

\section{Behavioral Results}

In the scanner, subjects were more accurate on object judgments compared to action judgments $[t(7)=2.9$, $p=.02]$, and they were faster on object judgments compared to action judgments $[t(7)=-5.1, p=.001]$ (see Table 1).

\section{Imaging Results}

Lateral temporal cortex. We used a region-of-interest (ROI) approach to test for activity differences in the lateral temporal cortex. First, anatomical ROIs were defined individually in each subject for the superior temporal, middle temporal, and inferior temporal gyrus. Then, within each ROI, we averaged the fMRI time series across all voxels that showed significant activity for the main effect of conceptual judgments compared to the baseline condition (see Figure 2). For each averaged time series, we calculated a measure of the difference in activity between the action and object conditions, and then tested whether this difference was significantly different from zero across subjects for each ROI (a random effects test, see Methods for details of all analyses). Because our previous findings for action words were limited to the left hemisphere and most of the activity for the main effect was in the left hemisphere, we focused our analyses on left hemisphere ROIs. Across subjects, activity was consistently greater for action judgments in the left middle temporal gyrus $[t(7)=4.0, p=.005]$ and in the left superior temporal gyrus $[t(7)=4.1, p=.005]$. Activity did not differ between the conditions in the left inferior temporal gyrus $[t(7)=0.3, p=.76$ ] (see Figure 3 and Table 2).

Table 1. Behavioral Performance (Mean \pm Standard Error) of Subjects in the fMRI Experiments for Different Task Conditions

Accuracy (\%) Reaction Time (msec)

Experiment 1

Actions

$87 \pm 2$

$1956 \pm 101$

Objects

$92 \pm 1$

$1802 \pm 94$

Baseline

$98 \pm 1$

$1201 \pm 60$

Experiment 2

Actions

$90 \pm 2$

$1994 \pm 86$

Manipulable Objects

$77 \pm 3$

$1984 \pm 101$

Animals

$81 \pm 3$

$2047 \pm 144$

Baseline

$98 \pm 1$

$1048 \pm 63$ 


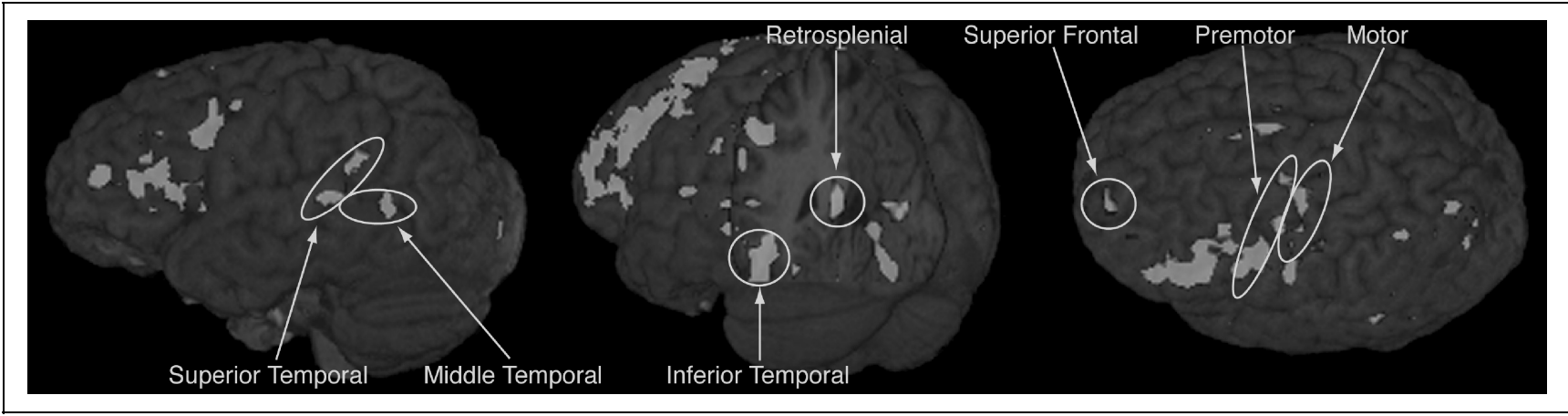

Figure 2. Example ROIs are shown. The main effect for the conceptual judgment task is displayed for three different subjects in Experiment 2 , with some ROIs for these subjects highlighted on the different views. ROIs were defined in each subject as all voxels significant for the main effect contrast within a specified anatomical area. Activity in these ROIs across both experiments is displayed in Figures 3 and 5.

$M T / M S T$. For each subject, we also defined an ROI for the MT/MST based on a localizer scan. Because there was little activity for the main effect in the MT/MST, we averaged across all ROI voxels in both hemispheres.
Across subjects, there was no consistent difference between action judgments and object judgments $[t(7)=$ $0.9, p=.39]$. This pattern did not change if we considered only voxels in the left hemisphere.

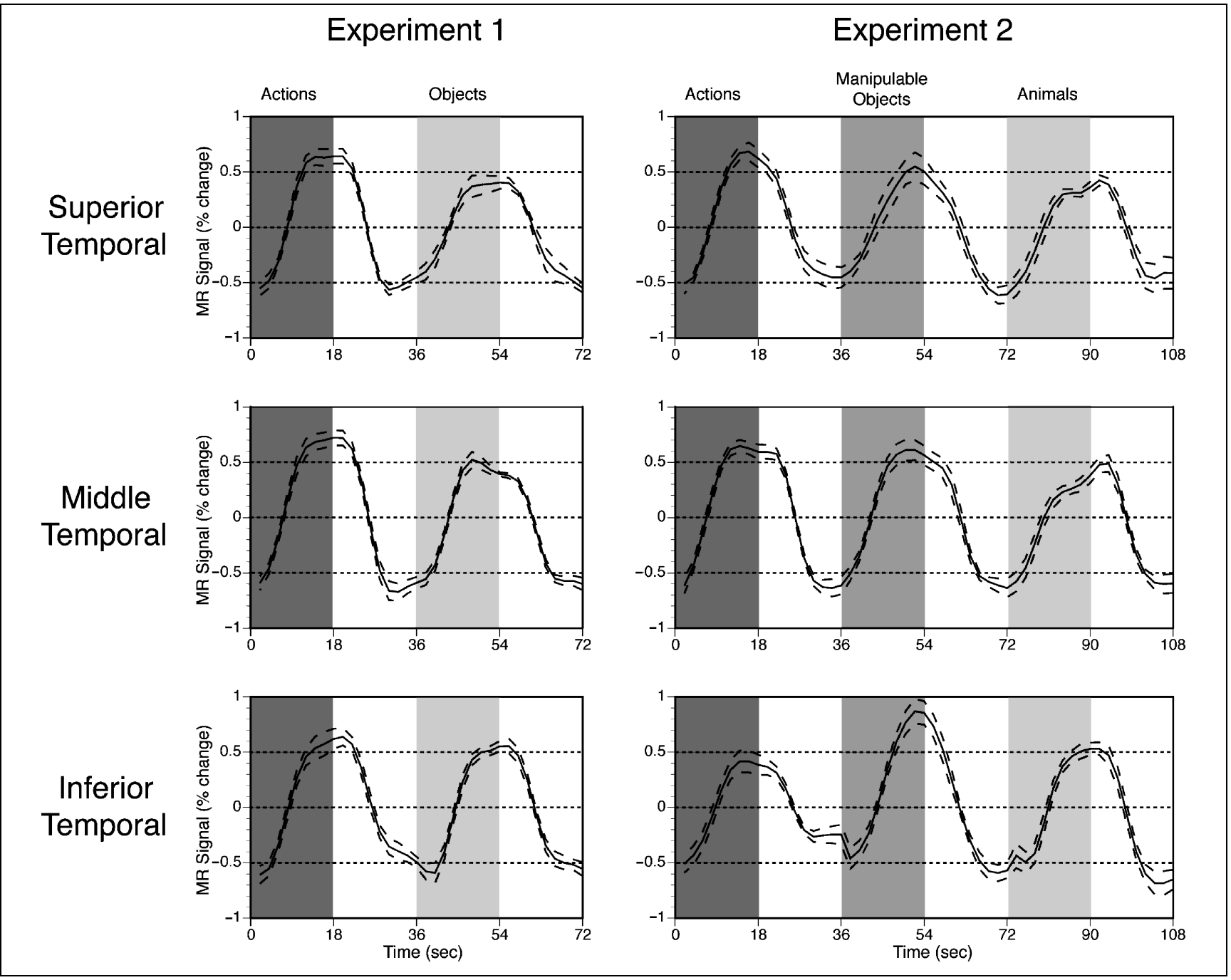

Figure 3. Displayed are averaged time series (and standard error) for lateral temporal ROIs in Experiments 1 and 2 . Time series were averaged across all blocks for each subject and then across all subjects for each experiment. Solid line depicts the average; dotted lines show average plus or minus standard error (calculated across subjects). 
Figure 4. Plotted is the location of the voxel showing the peak difference within the lateral occipito-temporal cortex for each subject in the present study and a previous one (Kable, Lease-Spellmeyer, et al., 2002). Peak voxels are shown for five contrasts: action words minus object words in judgments with unrelated distractors (O, $n=6$, previous study), action pictures minus object pictures ( $\square, n=6$, previous study), action words minus object words in judgments with related distractors $(\bullet, n=8$, Experiment 1), action words minus animal words in judgments with related distractors (घ, $n=9$, Experiment 2), and

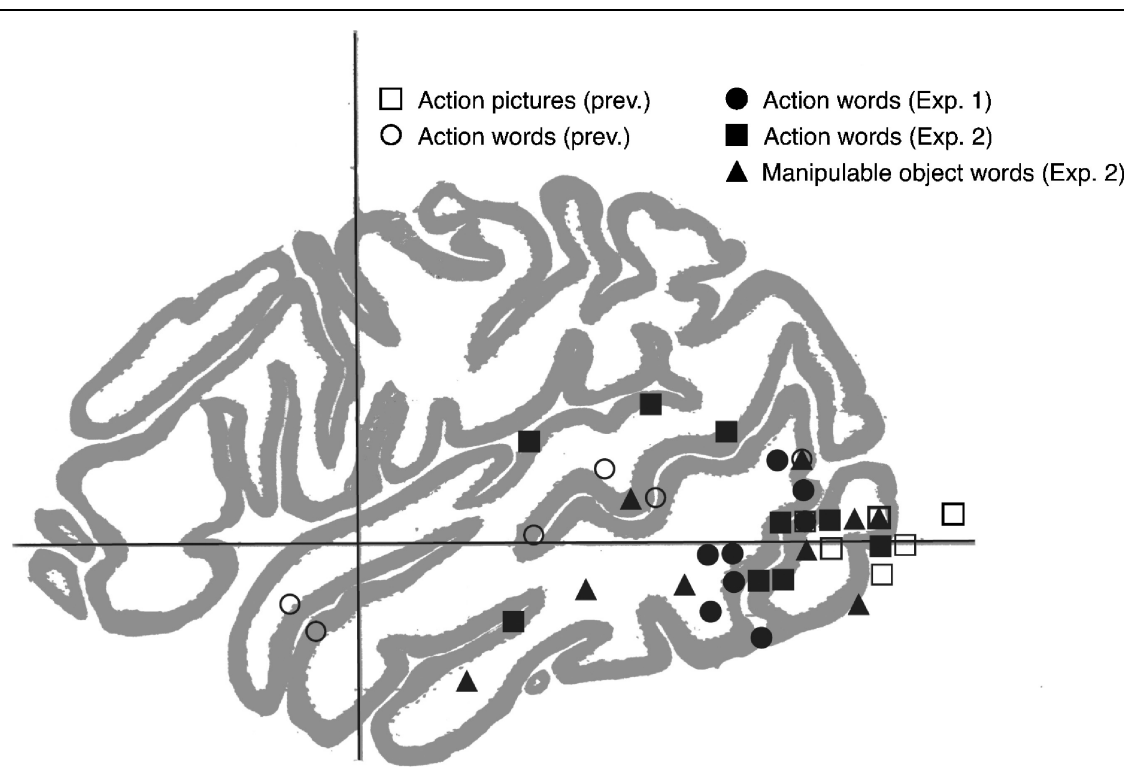

manipulable object words minus animal words in judgments with related distractors $(\boldsymbol{\Lambda}, n=9$, Experiment 2$)$. Locations are projected in the medial-lateral dimension onto a sagittal slice at $x=-51$. The most posterior peaks appear outside the brain for this projection because their true location is medial to this slice.

was intermediate between the two and not significantly different from either. In the left middle temporal gyrus, both action judgments and manipulable object judgments caused significantly greater activity than animal judgments [actions vs. animals, $t(8)=2.9, p=.02$; manipulable objects vs. animals, $t(8)=2.7, p=.03$ ]. In the left inferior temporal gyrus, activity for manipulable object judgments was significantly greater than both action judgments and animal judgments [actions vs. manipulable objects, $t(8)=-3.0, p=.02$; manipulable objects vs. animals, $t(8)=2.9, p=.02$ ] (see Figure 3 and Table 2 ).

Comparison of peak activity. In the same manner as the first experiment, we calculated the mean location (and standard error) of the peak difference within the left lateral occipito-temporal cortex between actions and animals $(-56 \pm 3,-54 \pm 6,1 \pm 4)$ and between manipulable objects and animals $(-53 \pm 2,-56 \pm 7$, $-6 \pm 4)$. The location of these two peaks was not significantly different. However, both peaks were more anterior than the peak for action pictures in the previous study [action words and pictures from the previous study, $t(13)=2.9, p=.01$; manipulable object words and pictures from the previous study, $t(13)=2.3$, $p=.04]$ and more posterior than the peak for action words in the previous study [action words and words from the previous study, $t(13)=-2.2, p=.049$; manipulable object words and words from the previous study, $t(13)=-2.2, p=.049$ ] (see Figure 4).

\section{Other Left Hemisphere Regions}

Although the main focus of our investigation was on the pattern of activity in the lateral occipito-temporal cortex, we also looked for activation differences outside of this region. To have similar sensitivity, we wanted to use the same ROI approach. In order to identify potential ROIs, we first performed an exploratory whole-brain analysis in normalized space on the data from the first experiment. Because the purpose of the exploratory analysis was to generate hypotheses, we used a liberal height threshold of $p<.01$ (uncorrected) and extent threshold of 5 voxels. At this threshold, there were eight clusters with greater activity for actions: seven were in the lateral temporal cortex and one was near the border of the ventral premotor and motor cortex (MNI coordinates of peak voxel: $-52.5,-7.5,15)$. There were also 10 clusters with greater activity for objects: 3 in the anterior portions of the superior frontal gyrus $(-7.5$, $52.5,-10 ; 15,26.25,40$; and $-30,15,35) ; 1$ in the lateral orbital gyrus $(-22.5,26.25,-20) ; 2$ in the fusiform gyrus $(-33.75,-48.75,-35$; and $22.5,-45,-25) ; 1$ in middle occipital gyrus $(-45,-78.75,15) ; 1$ in the inferior parietal cortex $(33.75,-86.25,35)$; and 2 extending from the posterior cingulate into the visual cortex $(3.75,-63.75,0$; and $-3.75,-75,10)$. Based on these findings, we decided to define additional anatomical ROIs in each subject in eight left hemisphere regions: lateral orbital gyrus, premotor cortex, motor cortex, superior frontal gyrus, fusiform gyrus, inferior parietal cortex, middle occipital gyrus, and retrosplenial cortex (see Methods for details on the anatomical criteria for these ROIs). For each of these new ROIs, we performed the same analysis as the one used in the lateral temporal ROIs.

Because the data from first experiment were used in the exploratory whole-brain analysis, the ROI analyses 
for these data are not an independent test. However, the exploratory analysis did not use a corrected significance threshold, so these ROI analyses do serve to confirm whether apparent differences in the exploratory analysis are significant. More importantly, the ROI analyses for the second experiment are independent from the hypothesis-generating whole-brain analysis. Therefore, consistent results across the two experiments would provide inferentially sound evidence for activation differences in an ROI.

In the first experiment, there was greater activity for action judgments in the left motor cortex $[t(7)=$ $2.8, p=.03]$, and a trend in the same direction that missed significance in the left premotor cortex $[t(7)=$ 2.3, $p=.055]$. The left retrosplenial cortex showed the opposite pattern, with consistently greater activity for object judgments compared to action judgments across subjects $[t(7)=-3.6, p=.009]$. In addition, increased activity for object judgments reached significance in the left superior frontal gyrus $[t(7)=$ $-3.0, p=.02]$, the left orbital gyrus $[t(7)=-2.5$, $p=.04]$, and the left middle occipital gyrus $[t(7)=$ $-2.5, p=.04]$. In the left fusiform and inferior parietal ROIs, we detected no significant differences between the action and object conditions (see Figure 5 and Table 2).

In the second experiment, these differences were only replicated in the retrosplenial and superior frontal ROIs, which again showed greater activity for objects compared to actions. In the left retrosplenial cortex, there was greater activity for both animal and manipulable object judgments compared to action judgments [actions vs. animals, $t(8)=-3.2, p=.01$; actions vs. manipulable objects, $t(8)=-2.9, p=.02]$. In the left superior frontal gyrus, only animal judgments showed greater activity than action judgments [actions vs. animals, $t(8)=-3.2, p=.01]$. In addition to these two replications, there was greater activity for manipulable object judgments compared to animal judgments in the left motor cortex [manipulable objects vs. animals, $t(8)=$ $3.1, p=.01]$, and a trend in the same direction in the left premotor cortex [manipulable objects vs. animals, $t(8)=2.1, p=.07]$. However, action judgments were not significantly different from either animal or manipulable object judgments in the left motor and premotor cortex. We also detected no significant differences in the lateral orbital or middle occipital ROIs. In the left fusiform gyrus, manipulable object judgments showed greater activity than action judgments, and trended towards greater activity than animal judgments [actions vs. manipulable objects, $t(8)=-3.1, p=.01$; manipulable objects vs. animals, $t(8)=-2.3, p=.052$ ] In the left inferior parietal cortex, activity was greater for both manipulable objects and animals compared to actions [actions vs. animals, $t(8)=-2.8, p=.02$; actions vs. manipulable objects, $t(8)=-2.5, p=.04$ ] (see Figure 5 and Table 2).

\section{Right Hemisphere Regions}

As explained above, we focused our analyses on left hemisphere ROIs, because our previous findings (and current hypotheses) concerned the left hemisphere. In addition, because our stimuli were words, we did not expect much activity in the right hemisphere a priori. Indeed, there was much less activity for the conceptual judgment task in right hemisphere areas-for example, the number of voxels significant for the main effect in the right middle temporal gyrus was 20\% the number of voxels in the left middle temporal gyrus. When we used the same approach to define the corresponding right hemisphere ROIs to those used above, there were only a few significant findings across the two experiments. In the first experiment, there was greater activity for action words compared to object words in the right middle temporal gyrus $[t(7)=2.4$, $p=.048]$. The right middle temporal gyrus showed a similar trend (greater activity for actions compared to animals) in the second experiment that did not reach significance $[t(8)=1.8, p=.10]$. Also in the second experiment, there was greater activity in the right fusiform gyrus for tools compared to actions $[t(8)=2.7$, $p=.025]$, and for animals compared to actions $[t(8)=$ $2.9, p=.02]$.

\section{DISCUSSION}

This study continues our investigations of the role of the lateral temporal cortex in mediating conceptual knowledge of action. In the first experiment, we replicated our previous finding that conceptual judgments of action words activate left posterior temporal regions close to, but not identical with, area MT/MST, under revised conditions where these judgments stressed detailed distinctions between visual motion features. In the second experiment, we extended these results by demonstrating that judgments of both action and manipulable object words caused greater activity than animal judgments in the middle temporal gyrus. Thus, semantic differences, rather than lexical or sublexical factors, seem to drive activation in the middle temporal gyrus. Finally, across both experiments, we found greater activity for object judgments in two unanticipated regions, the retrosplenial cortex and the superior frontal gyrus. We start by discussing our findings on the role of the lateral occipito-temporal cortex in verbally mediated conceptual knowledge, before briefly touching on our findings regarding other regions.

\section{Lateral Occipito-temporal Cortex}

In our first experiment, we tested the whether lateral occipito-temporal cortex would demonstrate greater 


\section{Experiment 1}
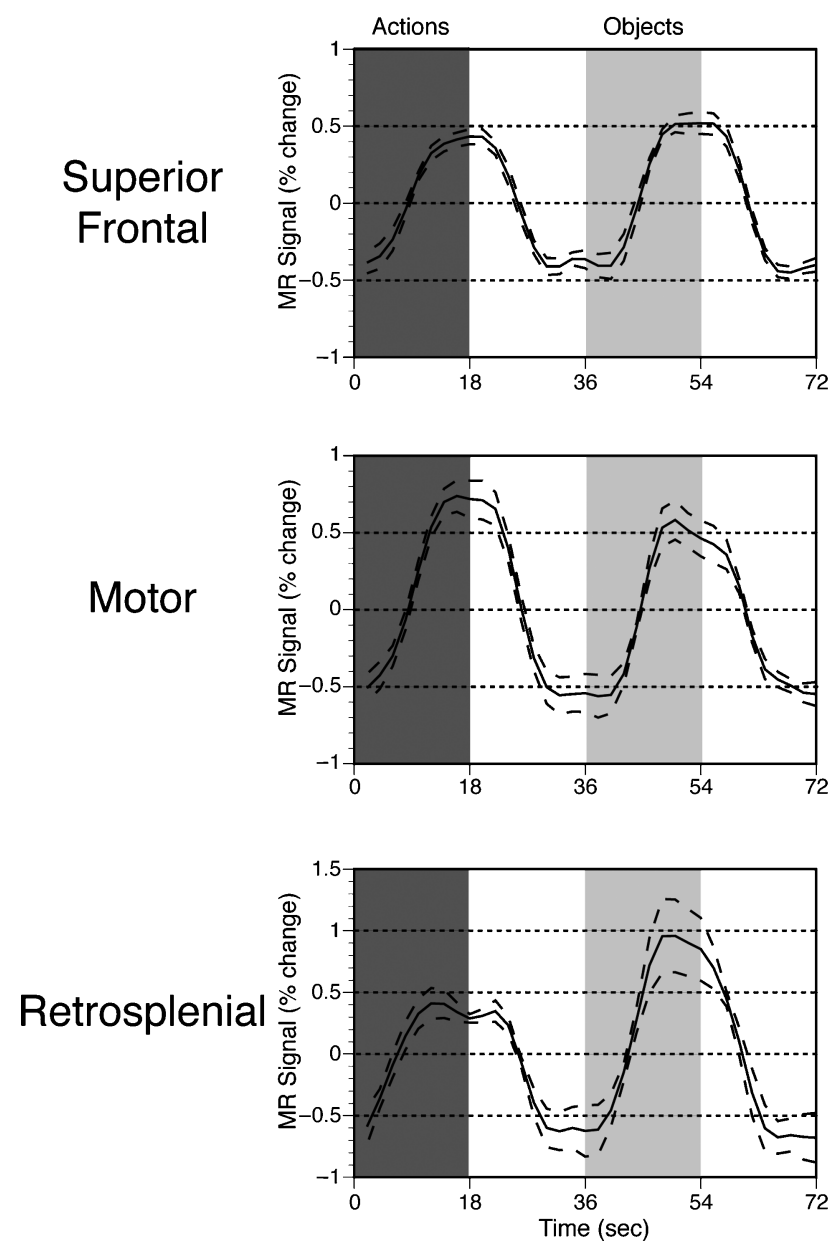

\section{Experiment 2}
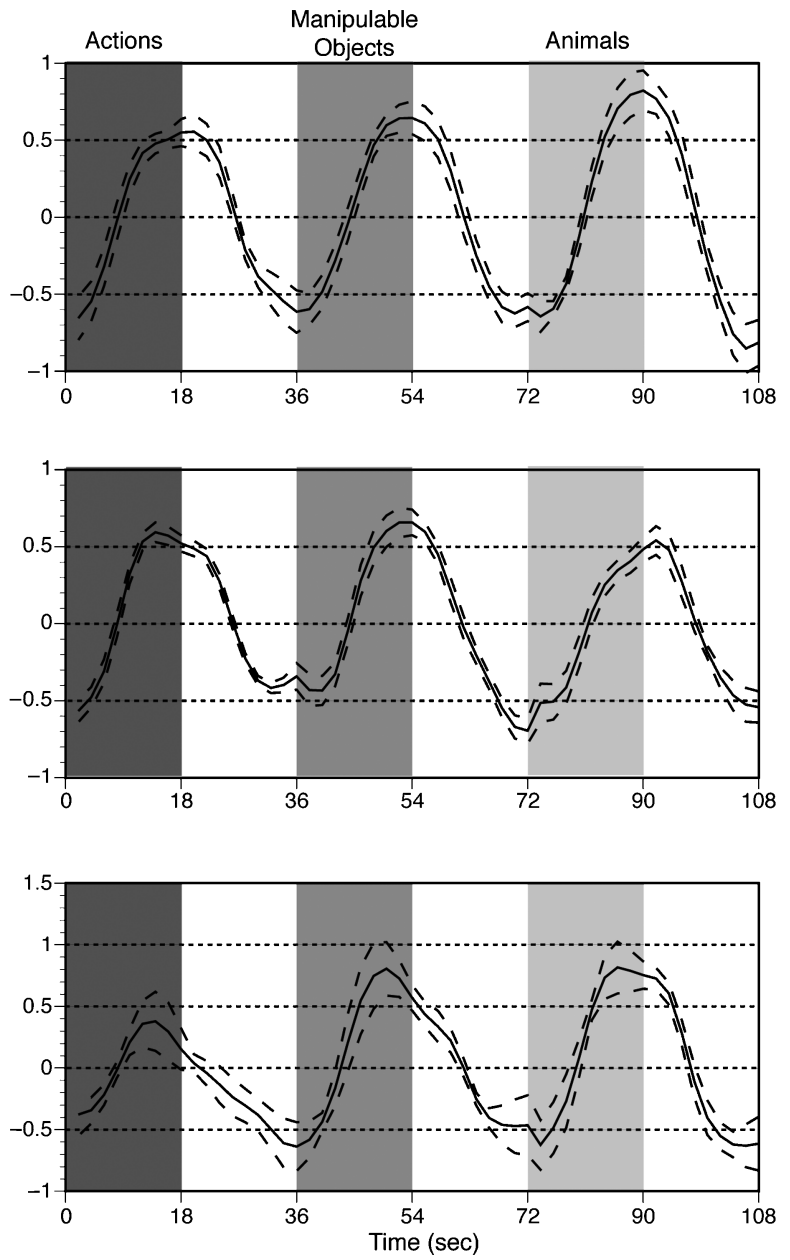

Figure 5. Displayed are averaged time series (and standard error) for other ROIs showing consistent effects in Experiments 1 and 2 . Time series were averaged across all blocks for each subject and then across all subjects for each experiment. Solid line depicts the average; dotted lines show average plus or minus standard error (calculated across subjects).

activity for action words than for object words in a task that stressed detailed distinctions between visual motion features. Under these conditions, action words elicited greater activity than object words in the left middle and superior temporal gyri. Action words, however, did not activate the MT/MST more than object words. These results replicate our previous findings that action words cause greater activity in left-lateralized posterior temporal regions, but not within the MT/MST (Kable, LeaseSpellmeyer, et al., 2002). They are also consistent with our suggestion for a gradient of motion representations within the lateral occipito-temporal cortex, such that areas processing verbal descriptors of motion activate regions close to, but not identical with, areas that mediate motion perception.

In our second experiment, we extended these findings by including two categories of object words, animals and manipulable objects. Again, action judgments elicited greater activity than animal judgments in the middle and superior temporal gyri on the left. Manipulable object judgments caused greater activation than animal judgments in the middle temporal gyrus, and greater activation than both action and animal judgments in the inferior temporal gyrus. This pattern of results supports the argument that semantic factors, rather than grammatical class, drives activation within the middle temporal gyrus.

Thus, the results across both experiments support the hypothesis that middle temporal gyrus activity is associated with semantic processing. Because activity in the middle temporal gyrus cuts across grammatical category boundaries, these findings are more consistent with theories that conceptual knowledge is mediated by a distributed network partially organized according to sensorimotor attributes, rather than theories positing conceptual organization along strict category lines. Indeed, our neural data show a similar pattern to one demonstrated in recent computational models of fea- 
ture-based semantic networks, where the clusters of conceptual similar items in self-organizing maps cross grammatical category boundaries (Vinson \& Vigliocco, 2002). Within such a feature-based network, the middle temporal gyrus may be particularly involved in the semantic processing of motion information, which would play a central role in conceptual representations of actions and manipulable objects.

A range of previous findings are consistent with these suggestions about the role of the middle temporal gyrus. In a set of studies similar to ours, Tyler and colleagues found greater activity in the lateral temporal cortex for judgments about biological actions compared to animal judgments (Tyler, Stamatakis, et al., 2003), but not for all verb judgments compared to all noun judgments (a category that included manipulable objects) (Tyler, Stamatakis, et al., 2003; Tyler, Russell, et al., 2001). The lateral temporal cortex also demonstrates greater activity when subjects generate action words compared to color words (Martin et al., 1995). Furthermore, damage in this area is associated with impairments on tests of conceptual knowledge for actions (Tranel, Kemmerer, Adolphs, Damasio, \& Damasio, 2003). In addition, activity in this region is found in a variety of tasks for tool words compared to animal words (Chao, Haxby, \& Martin, 1999; Perani, Schnur, et al., 1999; Thompson-Schill, Aguirre, D'Esposito, \& Farah, 1999; Cappa, Perani, Schnur, Tettamanti, \& Fazio, 1998), and damage in this area is associated with both impaired knowledge and impaired naming of tool pictures (Tranel, Damasio, \& Damasio, 1997; Damasio, Grabowski, Tranel, Hichwa, \& Damasio, 1996). Few previous studies, however, have directly compared actions and manipulable objects. Thus, our finding that action and manipulable object judgments cause distinct activations in the temporal cortex, which overlap in the middle temporal gyrus, provides important new evidence concerning the role of the lateral temporal cortex in conceptual processing.

Interpretation of the distinct areas of activity for actions and manipulable objects is less clear. One possibility is that these two categories are associated with diverging motion features-action words may more strongly evoke articulated, biological motion, whereas manipulable objects may more strongly evoke unarticulated, nonbiological motion. Consistent with this idea, Beauchamp, Lee, Haxby, and Martin (2002, 2003) directly compared pictures of moving tools with moving bodies and found greater activation for moving bodies in the superior temporal sulcus and greater activation for moving tools in the inferior temporal sulcus. Tranel, Martin, et al. (in press) found a similar overall pattern in the lateral temporal cortex for naming of actions and tools, with activation for actions more dorsal and tools more ventral. Our current results with words follow this same dorsal-ventral pattern. However, we cannot rule out possible alternative explanations. In particular, ac- tivity in the superior temporal gyrus may be based on lexical or sublexical factors, such as word length or grammatical class, especially considering the proximity of this activity to areas of the supramarginal gyrus implicated in phonological processing.

In the first experiment, we confirmed that activation for action words encompassed the posterolateral temporal cortex, but not the human MT/MST. In contrast, previous experiments with pictures have found greater activation for action pictures compared to object pictures that extends more posteriorly and includes the MT/MST (Kable, Lease-Spellmeyer, et al., 2002; Damasio, Grabowski, Tranel, Ponto, et al., 2001; Peigneux et al., 2000). Furthermore, Martin et al. (1995) observed a similar anterior shift for words compared to pictures in a verb generation task. These anterior-posterior differences between words and pictures could be evidence of a gradient of motion information represented in the occipito-temporal cortex, with areas closer to the MT/ MST representing more concrete visual information and areas closer to the middle temporal gyrus representing more abstract propositional information. Consistent with this proposal, the peak of greater activation for action (and manipulable object) words in the present two experiments was posterior to the peak of greater activation for action words in our previous study. Because the current task used related distractors to focus subjects' attention on the type of motion involved in different actions, subjects in the current study may have recruited comparatively more concrete visual information to perform the task. Furthermore, given this posterior shift, we would predict that there would be a greater overlap between the areas activated by action words and action pictures in the conceptual judgment task with related distractors, compared to the little overlap we observed previously with unrelated distractors (Kable, Lease-Spellmeyer, et al., 2002), as the related distractor task constrains subjects to use similar kinds of semantic information with both formats.

In both experiments, the difference between action (or manipulable object) and animal words in the middle temporal gyrus was smaller than the difference between any of the conceptual conditions and the perceptual baseline. As can be seen in Figure 3, there was activation in the lateral temporal cortex for the animal judgments compared to the perceptual baseline task. However, interpreting this activation, or any activation referenced to the baseline, is difficult because the perceptual baseline task was not intended to be a precise control for the conceptual judgment task (see Methods). Thus, much of the difference between animal judgments and the baseline perceptual matching task may be explained by nonspecific factors such as attention or task difficulty. That is not to say that knowledge of animals might not be informed by motion attributes. Rather, our claim is that motion attributes 
make a relatively greater contribution to action and manipulable object knowledge compared to animal knowledge.

\section{Other Regions}

\section{Left Frontal Cortex}

In the first experiment, activity in the left motor cortex was consistently greater for action judgments compared to object judgments. In the second experiment, we did not replicate this effect in the left motor cortex, although we did find greater activity for manipulable object judgments compared to animal judgments. We also failed to find differences in motor or premotor areas between action and object judgments in our previous study. This pattern of results provides weak support for the idea that motor and premotor areas are important for conceptual representations of action.

In contrast to our results, many previous studies have found an association between action-naming difficulties and left frontal damage (Hillis, Tuffiash, Wityk, \& Barker, 2002; Tranel, Adolphs, Damasio, \& Damasio, 2001; Daniele et al., 1994; Damasio \& Tranel, 1993), or increased activity in the left frontal cortex for tool naming compared to animal naming (Chao \& Martin, 2000; Grabowski, Damasio, \& Damasio, 1998; Grafton, Fadiga, Arbib, \& Rizzolatti, 1997; Perani, Cappa, Bettinardi, et al., 1995). However, these findings could be explained by processes other than action semantics that have been associated with the left frontal cortex. For example, the left frontal cortex has been associated with the morphological processing of verbs (Tyler, Bright, et al., 2004; Shapiro \& Caramazza, 2003), which could explain actionnaming deficits. Differences in naming associated with the left frontal cortex could also be due to more general processes, such as selection from semantic memory (Thompson-Schill, 2003; Thompson-Schill, D'Esposito, \& Kan, 1999; Thompson-Schill, Swick, et al., 1998; Thompson-Schill, D'Esposito, Aguirre, \& Farah, 1997), that might be particularly stressed for certain categories of stimuli. Alternatively, the left frontal cortex may participate in representations of motor programs associated with different concepts (Hauk, Johnsrude, \& Pulvermuller, 2004; Kellenbach, Brett, \& Patterson, 2003; Kraut, Moo, Segal, \& Hart, 2002; Mecklinger, Gruenewald, Besson, Magnie, \& Von Cramon, 2002; Rizzolatti, Fogassi, \& Gallese, 2001), but these motor attributes may play a less central role than sensory ones in the representation of action concepts generally, or at least for the specific stimuli we used. In addition, these different processes may explain effects in different parts of the left frontal cortex. Along these lines, we recently found a dissociation between activity during naming in the left ventral premotor cortex, which was associated with manipulability, and in the left inferior frontal gyrus, which was associated with se- lection demands (Kan, Kable, van Scoyoc, Chatterjee, \& Thompson-Schill, in press).

\section{Ventral Occipito-temporal Cortex}

If the lateral occipito-temporal cortex plays an important role in action representations, then one might expect the ventral occipito-temporal cortex to play a similar role in object representations. Consistent with this notion, we found greater activation for manipulable objects compared to actions in inferior temporal and fusiform areas. However, we only found greater activation for animal judgments compared to actions in the right fusiform in the second experiment. In both the first experiment and a previous study, we failed to find greater activation for animal judgments in ventral occipito-temporal areas. One explanation for these inconsistent findings may be that action concepts cannot be accessed independently of the actors or objects involved in the action. Consequently, one would predict that object-specific areas in the ventral occipito-temporal cortex would not respond differentially depending on whether the object was participating in an action. This was exactly the pattern reported in a recent experiment by Beauchamp et al. (2002). Ventral occipito-temporal areas showed object-specific responses, but these responses were not modulated by whether the object was moving, whereas lateral occipito-temporal areas showed greater responses for moving compared to stationary stimuli.

\section{Retrosplenial Cortex}

Across both experiments, we found consistently greater activity for object words in two unanticipated regions, one of which was the left retrosplenial cortex. In the second experiment, left retrosplenial activity was greater for both animals and manipulable objects compared to actions. The retrosplenial cortex has previously been associated with episodic memory processing. Damage to the retrosplenial cortex is associated with memory deficits (Valenstein et al., 1987), and damage to the left retrosplenial cortex is associated with verbal memory deficits (McDonald, Crosson, Valenstein, \& Bowers, 2001). In addition, functional imaging studies find retrosplenial activation associated with episodic encoding (Shallice et al., 1994). In both experiments of the current study, the object words were more concrete than the action words, and concrete words are better remembered than abstract words. Thus, one possible interpretation of our results is that left retrosplenial activity is related to encoding words into episodic memory, a process unrelated to performing the conceptual judgment task. Consistent with this explanation, subjects performing the same conceptual judgment tasks used in the two current experiments remember more object 
words from the task than action words in a free recall paradigm (data not shown).

\section{Superior Frontal Cortex}

The superior frontal cortex was the second unanticipated region showing greater activity for objects compared to actions. In the second experiment, the superior frontal cortex showed greater activity only for animals compared to actions. Previously, activity was found in portions of the anterior superior frontal gyrus during a lexical decision task, as well as during other tasks thought to involve accessing word meaning (Binder et al., 2003). A nearby region of the superior frontal gyrus shows greater activity during naming of unique persons (Damasio, Tranel, et al., 2004) or judgments about persons (Mitchell, Heatherton, \& Macrae, 2002). Given this pattern of results, the anterior superior frontal cortex might be involved in conceptual representations of animate objects.

\section{Conclusion}

Accessing knowledge of actions and manipulable objects causes distinct activations in the posterior lateral temporal cortex, which overlap in the middle temporal gyrus. These findings are compatible with the general view that conceptual knowledge is instantiated by distributed neural regions partially organized along sensorimotor lines. Specifically, these data support the idea that the lateral temporal cortex is important in mediating representations of motion features central to action concepts. Furthermore, we suggest that different motion representations are organized along a concrete to abstract gradient, which extends from the occipitotemporal junction into the middle temporal gyrus and peri-sylvian cortices.

\section{METHODS}

\section{Subjects}

Eight right-handed individuals participated in the first experiment ( 4 women, 4 men, mean age $=23$ years). A ninth subject was excluded from the study because of excessive head movement in the scanner $(>2 \mathrm{~mm}$ across the scanning session). Nine right-handed individuals participated in the second experiment ( 5 women, 4 men, mean age $=21$ years). Given that sex differences have previously been reported during language tasks (Grabowski, Damasio, Eichhorn, \& Tranel, 2003), we tried to balance the number of men and women in each experiment. All subjects were recruited from the university community, and all spoke only English before school age. None had a history of neurologic or psychiatric symptoms. All subjects gave informed consent in accordance with the procedures of the Institutional Review Board of the University of Pennsylvania.

\section{Behavioral Tasks}

In both experiments, subjects performed a conceptual similarity judgment task with words during scanning (see Figure 1). In each trial, subjects were presented with three words, one at the top of the screen and two at the bottom. Subjects pressed a button (right or left hand) to indicate which of the two words at the bottom was more closely related in meaning to the word at the top. In baseline trials, subjects were presented with three false font strings, and they indicated which of the two at the bottom of the screen was the same as the one on top.

The stimuli from the first experiment consisted of 40 action word triads and 40 object word triads. All three words in a triad referred to actions or objects from the same general category. Action categories included manners of motion, paths of motion, and movements of different body parts. Object categories were restricted to animals, fruits and vegetables, trees, flowers, and food. The stimuli for the second experiment consisted of 32 action word triads, 32 animal word triads, and 32 manipulable object word triads. Manipulable objects included the categories of tools, kitchen utensils, cleaning implements, office implements, and weapons. Because the second experiment was partially designed to test the effect of grammatical category, words that could refer to both a tool and the use of that tool (e.g., "hammer") were not used in either the action or manipulable object categories. Given that English words can frequently be used as both nouns and verbs, it would have been impossible to exclude all noun-verb homonyms. However, the context of comparing three related words, as well as the consistent ending of action words, served to constrain subjects' interpretations of words as denoting objects or actions.

Because in all cases the three words in a triad were from the same category, they tended to share several semantic attributes, and the choice was usually distinguished on the basis of one salient feature. Using three words from the same category also reduced the importance of syntactic information in making judgments about action words. Although the syntactic frames of a verb constrains verb meaning, this information best distinguishes different categories of actions (Fisher, Gleitman, \& Gleitman, 1991). For example, motion verbs like "walk" and "slide" can both occur in sentences with one noun argument and a prepositional phrase, whereas cognition verbs like "think" and "believe" can both occur in sentences with one noun argument and a sentential complement.

Triads were selected from a larger initial set on the basis of pilot testing so that response agreement (the proportion of subjects choosing a particular response) 
and reaction time were similar across conditions. We tested 11 pilot subjects for the first experiment ( 9 women, 2 men, mean age $=27$ years) and 12 pilot subjects for the second experiment ( 5 women, 7 men, mean age $=19$ years). Equal numbers of left and right responses were chosen in each condition. No triads were repeated, although individual words could be repeated once. Because many English words can refer to actions or objects, all action words were presented in the present participle form, ending in "-ing." Although this consistent ending might have created a potential confound (by making action words longer, for example), we felt it was necessary to ensure that subjects understood the words as clearly referring to actions. Baseline trials were created by replacing one of the choice words with the top word and changing all three words to strings in Wingdings font.

We validated these tasks in a second pilot study with 24 right-handed subjects (20 women, 4 men, mean age $=21$ years). Twelve subjects performed the conceptual similarity judgment task from the first experiment, and 12 performed the task from the second experiment. In addition, because eye movements can cause differences in neural activity (Barton et al., 1996), we recorded eye position using an iView RED-II camera system (Sensomotoric Instruments, Boston, MA, www.smi.de) to verify that eye movements did not systematically differ across conditions. For each condition, we measured subjects' accuracy, reaction time, and number of fixations. Fixations, an index of the amount of eye movements, were defined as gaze position remaining with a restricted area (radius less than approximately $2 \mathrm{~mm}$ ) for greater than $100 \mathrm{msec}$. These data are reported in Table 3. There were no differences across conditions in reaction time or number of fixations.

In evaluating subjects, we consider a response correct if it was the one chosen by the majority of pilot subjects. In this context, subjects could make an "incorrect"

Table 3. Results (Mean \pm Standard Error) of Pilot Study for Stimuli Used in Experiments 1 and 2

\begin{tabular}{lccc}
\hline & $\begin{array}{c}\text { Total } \\
\text { Fixations }\end{array}$ & $\begin{array}{c}\text { Accuracy } \\
(\%)\end{array}$ & $\begin{array}{c}\text { Reaction Time } \\
(\text { msec })\end{array}$ \\
\hline $\begin{array}{l}\text { Experiment } 1 \\
\text { Actions }\end{array}$ & $8.46 \pm 0.36$ & $93 \pm 1$ & $1710 \pm 77$ \\
Objects & $8.35 \pm 0.44$ & $92 \pm 1$ & $1702 \pm 73$ \\
& & & \\
Experiment 2 & & & \\
Actions & $8.03 \pm 0.21$ & $90 \pm 2$ & $1907 \pm 72$ \\
Manipulable Objects & $7.88 \pm 0.22$ & $84 \pm 2$ & $1984 \pm 78$ \\
Animals & $7.79 \pm 0.21$ & $88 \pm 2$ & $1913 \pm 80$ \\
\hline
\end{tabular}

choice for valid reasons, based on a semantic property that choice shares with the target. For our purposes, it was only important that subjects engaged in the task and accessed semantic information in making their judgment, which seemed likely given their $~ 90 \%$ accuracies in each condition.

We decided to choose stimuli on the basis of reaction time, rather than on various lexical variables. Values of these different variables are provided in Table 4. We prioritized reaction time because it could clearly have an effect on neural activity, as the fMR response is sensitive to the length as well as amount of neural activity. As already mentioned, action words were longer because of the morphological ending, and verbs are systematically rated lower in concreteness and imageability. Previous investigators have noted this confounding of conceptual categories with lexical variables, and argued that matching can result in idiosyncratic stimulus sets (Tranel, Adolphs, et al., 2001). However, we are sensitive to the possibility that these variables may affect activation, which forms part of the motivation for testing multiple categories in the second experiment. Because the manipulable object words are similar to animals in length, concreteness, and imageability, activation for both actions and manipulable objects rules out these potential confounding explanations.

In the first experiment, subjects also participated in a scan to functionally localize the MT/MST. In this scan, subjects passively viewed alternating 16-sec blocks of radially moving rings and stationary rings. The radially moving rings alternated between inward and outward motion every $2 \mathrm{sec}$.

\section{Stimulus Presentation}

Stimuli were presented to the subjects using a highresolution, fiber-optic presentation system carrying information from an external computer to goggles attached to the head coil (Avotec, Stuart, FL, www.avotec.org). Timing of stimulus presentation was synchronized with the scanner, so that a new stimulus was always presented at the beginning of a repetition. Subject responses were transmitted from the scanner room using a customdesigned, fiber-optic, motor response-recording device (Current Design Concepts, Philadelphia, PA). Stimulus presentation and recording of responses were controlled by Psyscope software (psyscope.psy.cmu.edu) for the conceptual tasks and Pixx software (psychology. concordia.ca/department/CVLab/CVLab.html) for the visual motion scan.

\section{MRI Acquisition}

BOLD-sensitive, T2*-weighted fMRI data were acquired on a 1.5-T GE Signa scanner using a gradient-echo, echoplanar pulse sequence $\left(\mathrm{TR}=2000 \mathrm{msec}, \mathrm{TE}_{\mathrm{eff}}=\right.$ 
Table 4. Values of Psycholinguistic Variables for the Words Used in Each Condition

Letter Length Syllable Length Log Frequency Familiarity Concreteness Imageability

Experiment 1

$\begin{array}{lllllll}\text { Actions } & 7.8 \pm 0.1(120) & 2.1 \pm 0.03(120) & 1.12 \pm 0.06(108) & 529 \pm 6(69) & 487 \pm 10(64) & 513 \pm 8(68) \\ \text { Objects } & 5.5 \pm 0.2(120) & 1.7 \pm 0.1(120) & 0.88 \pm 0.06(86) & 509 \pm 6(82) & 602 \pm 3(82) & 593 \pm 4(82)\end{array}$

Experiment 2

\begin{tabular}{lllllll} 
Actions & $7.8 \pm 0.1(96)$ & $2.1 \pm 0.03(96)$ & $0.99 \pm 0.07(81)$ & $506 \pm 9(55)$ & $478 \pm 9(52)$ & $509 \pm 7(53)$ \\
Manipulable Objects & $5.6 \pm 0.2(96)$ & $1.6 \pm 0.1(96)$ & $0.97 \pm 0.08(67)$ & $505 \pm 8(70)$ & $579 \pm 6(61)$ & $563 \pm 6(61)$ \\
Animals & $5.8 \pm 0.2(96)$ & $1.8 \pm 0.1(96)$ & $0.70 \pm 0.07(61)$ & $476 \pm 9(64)$ & $608 \pm 3(58)$ & $595 \pm 5(58)$ \\
\hline
\end{tabular}

Values are from the MRC Psycholinguistic Database, and are reported as mean \pm standard error. Numbers in parentheses are the number of words for which that variable was listed in the database.

50 msec, flip angle $=90^{\circ}$ ). The scanner was equipped with a standard clinical quadrature radio-frequency head coil and a prototype gradient system (GESR230) capable of ultrafast imaging. Data were acquired in twenty-one 5-mm axial slices, covering the entire cortex but omitting lower portions of the cerebellum and brainstem. The resolution within each slice was $3.75 \times$ $3.75 \mathrm{~mm}(64 \times 64$ matrix within a $24-\mathrm{cm}$ field of view). Phase maps were acquired to correct for distortion in the echo-planar images. Head motion was minimized using foam padding. Subjects performed no task during the first $20 \mathrm{sec}$ of each scan as steady-state magnetization was achieved. Subjects in the first experiment completed two 6-min 20-sec scans while performing the conceptual judgment task, for a total of 360 observations per voxel per subject. Subjects in the second experiment completed two 7-min 32-sec scans while performing the conceptual judgment task, for a total of 432 observations per voxel per subject. For each subject, high-resolution, T1-weighted axial and sagittal scans were also acquired using a spin-echo sequence $\left(\mathrm{TR}=500 \mathrm{msec}, \mathrm{TE}=8 \mathrm{msec}\right.$, flip angle $\left.=90^{\circ}\right)$.

\section{Data Analysis}

\section{Statistical Tests}

Data processing was performed off-line using software developed at the University of Pennsylvania (www.voxbo.org). After reconstructing images from the raw data, data were sinc-interpolated in time to correct for staggered slice acquisition, realigned to the first image acquired for each subject using a six-parameter motion-correction algorithm, and thresholded to exclude extra-parenchymal voxels from subsequent analyses. Within each subject, a voxelwise analysis was performed using a version of the modified general linear model. Included in this model were covariates modeling different task conditions (e.g., "action judgments"), a subject-specific estimate of the intrinsic temporal autocorrelation, and sine and cosine regressors for frequencies below those of the task and for frequencies in the elevated range of the noise spectrum. Task covariates were boxcar waveforms convolved with an estimate of the BOLD hemodynamic transfer function empirically derived from the motor cortex in a large group of subjects (Aguirre, Zarahn, \& D'Esposito, 1998). To improve specificity, data were also smoothed in time with the hemodynamic transfer function. These methods have been empirically demonstrated to control the false-positive rate (Aguirre, Zarahn, \& D'Esposito, 1997; Zarahn, Aguirre, \& D'Esposito, 1997).

In the first experiment, reaction times for our two conditions of interest differed for the subjects participating in the fMRI experiment, even though they did not in our pilot testing. Because reaction time differences alone could account for activation differences, we also included in our statistical model regressors that covaried out effects of reaction time differences (Kable, Kimberg, \& Chatterjee, 2004). Using individual subject reaction times for each trial, we modeled effects of reaction time for the conceptual task (collapsed across action and object conditions) using orthogonal polynomial covariates (orthogonalized with respect to the main effect of the conceptual task) (Buchel, Holmes, Rees, \& Friston, 1998). Because reaction times were longer for action judgments than for object judgments, covarying for reaction time reduces the amount of activation for actions (and increases the amount for objects) in the direct contrast between these conditions.

We tested hypotheses about activity differences between conditions using an ROI approach. After defining ROIs, we performed a random-effects group analysis as follows. Initially, voxels were identified within each subject where activity was significant for the main effect of conceptual judgment compared to baseline conditions ( $p<.05$, Bonferroni-corrected for multiple comparisons within an ROI). Unlike in cognitive subtraction 
paradigms, the baseline condition was not designed to be closely matched to the conditions of interest. Rather, the main effect comparison simply reduced the number of voxels within which specific hypotheses about differential activity for the contrasts of interest were tested. The fMRI time series was averaged for all voxels significant for the main effect within a defined ROI in each subject. Next, a measure of the effect size for the orthogonal contrast between conditions of interest (e.g., actions minus objects in the first experiment or actions minus animals in the second experiment) was extracted from the spatially averaged ROI time series in each subject. We used $t$ values as a measure of effect size rather than percent signal change, because the residual error term in the denominator of the $t$ value most effectively corrects the effect size for scaling effects due to differences in overall MR signal intensity across scanning sessions (Postle, Zarahn, \& D'Esposito, 2000). Finally, paired $t$ tests addressed whether this effect was consistently greater for one condition or the other in that ROI across subjects. This analysis gains sensitivity by reducing the effect size in each ROI to one value and avoiding the need to correct for multiple comparisons across voxels, although it loses spatial resolution by averaging across all active voxels within an ROI.

We performed an additional analysis to compare the results from this study to those of our previous study. In particular, a shift in the location of activation within the lateral occipito-temporal cortex between the two studies might not be revealed by our ROI analyses. In order to look for such a shift, we derived unthresholded $t$-maps for the direct contrast of actions and objects in each subject. These $t$-maps were then normalized into the standard coordinate space of the MNI brain using a 12-parameter affine transformation with nonlinear deformations. Normalization parameters were initially calculated using each subject's high-resolution anatomical scan. After normalization, the maps were spatially smoothed with a kernel with full-width halfmaximum of $7.5 \mathrm{~mm}$. We found the location of the voxel with the peak difference between actions and objects in the lateral occipito-temporal cortex in each subject, and computed the average location in MNI coordinates of this peak across subjects. We performed a similar analysis on the data from our previous study, and used $t$ tests to compare the location of the peak difference in this study to the location of the peak differences (for both words and pictures) in our previous study.

We also used the normalized unthresholded $t$-maps from the first experiment in a hypothesis-generating whole-brain analysis. Specifically, we performed a random-effects analysis at each voxel by testing whether the difference between actions and object was significantly different from zero across subjects. Because this analysis was exploratory, we thresholded the results at a height threshold of $p<.01$ and an extent threshold of 5 voxels.

\section{Definition of ROIs}

The locations of anatomical ROIs were determined with reference to a combined MRI/anatomical atlas. Anatomical ROIs were traced on each subject's highresolution, T1-weighted image by one author and checked by a second author. The lateral orbital ROI included the lateral orbital gyrus, inferior to the inferior frontal ROI. The lateral premotor ROI corresponded to Brodmann's area 6 laterally, including the cortex surrounding the precentral sulcus below the superior frontal gyrus. The motor ROI included the precentral gyrus, excluding any areas in the premotor ROI. The superior frontal ROI included the anterior half of the superior frontal gyrus. The fusiform ROI included both the occipital and temporal portions of this gyrus. The inferior, middle, and superior temporal ROIs included the posterior two-thirds of these gyri. The inferior parietal ROI included the angular and supramarginal gyri, corresponding to Brodmann's areas 39 and 40. The middle occipital ROI included all of the middle occipital gyrus. The retrosplenial ROI included portions of the posterior cingulate cortex immediately surrounding the splenium.

For the first experiment, the MT/MST ROI was functionally identified in each subject in a separate localizer scan. In accordance with previous studies, we defined the visual motion cortex on the basis of the contrast between moving rings and stationary rings. We included in the MT/MST all significant voxels contiguous with the peak activation on the lateral occipital surface. This region appears to be the human homologue of motion-sensitive cortical areas in the macaque, including the MT and MST (Tootell et al., 1995; Watson et al., 1993). Within this group of subjects, both the size of the MT/MST ( $\sim 20$ voxels/hemisphere) and its location (within a sulcus near the junction of the inferior temporal sulcus and the ascending limb of the inferior temporal sulcus) were consistent with previous studies (Dumoulin et al., 2000).

\section{Acknowledgments}

This research was supported by an NSF graduate fellowship to J. K. and NIH grant R01 DC004817 to A. C. We thank Scott Greenwald for running some of the pilot experiments, and Geoff Aguirre, John Detre, and Dan Kimberg for discussions about issues involved in covarying for reaction time in fMRI analyses.

Reprint requests should be sent to Anjan Chatterjee, Department of Neurology, University of Pennsylvania, 3 West Gates Building, 3400 Spruce Street, Philadelphia, PA 19104-4283, or via e-mail: anjan@mail.med.upenn.edu. 
The data reported in this experiment have been deposited with the fMRI Data Center archive (www.fmridc.org). The accession number is 2-2005-118X8.

\section{REFERENCES}

Aguirre, G. K., Zarahn, E., \& D'Esposito, M. (1997). Empirical analyses of BOLD fMRI statistics: II. Spatially smoothed data collected under null-hypothesis and experimental conditions. Neuroimage, 5, 199-212.

Aguirre, G. K., Zarahn, E., \& D'Esposito, M. (1998). The variability of human, BOLD hemodynamic responses. Neuroimage, 8, 360-369.

Barton, J. J., Simpson, T., Kiriakopoulos, E., Stewart, C., Crawley, A., Guthrie, B., Wood, M., \& Mikulis, D. (1996). Functional MRI of lateral occipitotemporal cortex during pursuit and motion perception. Annals of Neurology, 40, 387-398.

Beauchamp, M. S., Lee, K. E., Haxby, J. V., \& Martin, A. (2002). Parallel visual motion processing streams for manipulable objects and human movements. Neuron, 34, 149-159.

Beauchamp, M. S., Lee, K. E., Haxby, J. V., \& Martin, A. (2003). FMRI responses to video and point-light displays of moving humans and manipulable objects. Journal of Cognitive Neuroscience, 15, 991-1001.

Binder, J. R., McKiernan, K. A., Parsons, M. E., Westbury, C. F., Possing, E. T., Kaufman, J. N., \& Buchanan, L. (2003). Neural correlates of lexical access during visual word recognition. Journal of Cognitive Neuroscience, 15, 372-393.

Buchel, C., Holmes, A. P., Rees, G., \& Friston, K. J. (1998). Characterizing stimulus-response functions using nonlinear regressors in parametric fMRI experiments. Neuroimage, 8 , 140-148.

Buxbaum, L. J., \& Saffran, E. M. (2002). Knowledge of object manipulation and object function: Dissociations in apraxic and nonapraxic subjects. Brain and Language, 82, 179-199.

Cappa, S. F., Perani, D., Schnur, T., Tettamanti, M., \& Fazio, F. (1998). The effects of semantic category and knowledge type on lexical-semantic access: A PET study. Neuroimage, 8, 350-359.

Caramazza, A., Hillis, A. E., Rapp, B. C., \& Romani, C. (1990). The multiple semantics hypothesis: Multiple confusions? Cognitive Neuropsychology, 7, 161-189.

Caramazza, A., \& Mahon, B. Z. (2003). The organization of conceptual knowledge: The evidence from category-specific semantic deficits. Trends in Cognitive Sciences, 7 , 354-361.

Chao, L. L., Haxby, J. V., \& Martin, A. (1999). Attribute-based neural substrates in temporal cortex for perceiving and knowing about objects. Nature Neuroscience, 2, 913-919.

Chao, L. L., \& Martin, A. (2000). Representation of manipulable man-made objects in the dorsal stream. Neuroimage, 12, 478-484.

Damasio, A. R., \& Tranel, D. (1993). Nouns and verbs are retrieved with differently distributed neural systems. Proceedings of the National Academy of Sciences, U.S.A., 90, 4957-4960.

Damasio, H., Grabowski, T. J., Tranel, D., Hichwa, R. D., \& Damasio, A. R. (1996). A neural basis for lexical retrieval. Nature, 380, 499-505.

Damasio, H., Grabowski, T. J., Tranel, D., Ponto, L. L., Hichwa, R. D., \& Damasio, A. R. (2001). Neural correlates of naming actions and of naming spatial relations. Neuroimage, 13, 1053-1064.

Damasio, H., Tranel, D., Grabowski, T., Adolphs, R., \&
Damasio, A. (2004). Neural systems behind word and concept retrieval. Cognition, 92, 179-229.

Daniele, A., Giustolisi, L., Silveri, M. C., Colosimo, C., \& Gainotti, G. (1994). Evidence for a possible neuroanatomical basis for lexical processing of nouns and verbs. Neuropsychologia, 32, 1325-1341.

Dumoulin, S. O., Bittar, R. G., Kabani, N. J., Baker, C. L., Jr., Le Goualher, G., Bruce Pike, G., \& Evans, A. C. (2000). A new anatomical landmark for reliable identification of human area V5/MT: A quantitative analysis of sulcal patterning. Cerebral Cortex, 10, 454-463.

Fisher, C., Gleitman, H., \& Gleitman, L. R. (1991). On the semantic content of subcategorization frames. Cognitive Psychology, 23, 331-392.

Gainotti, G. (2000). What the locus of brain lesion tells us about the nature of the cognitive defect underlying category-specific disorders: A review. Cortex, 36, 539-559.

Grabowski, T. J., Damasio, H., \& Damasio, A. R. (1998). Premotor and prefrontal correlates of category-related lexical retrieval. Neuroimage, 7, 232-243.

Grabowski, T. J., Damasio, H., Eichhorn, G. R., \& Tranel, D. (2003). Effects of gender on blood flow correlates of naming concrete entities. Neuroimage, 20, 940-954.

Grafton, S. T., Fadiga, L., Arbib, M. A., \& Rizzolatti, G. (1997) Premotor cortex activation during observation and naming of familiar tools. Neuroimage, 6, 231-236.

Hauk, O., Johnsrude, I., \& Pulvermuller, F. (2004). Somatotopic representation of action words in human motor and premotor cortex. Neuron, 41, 301-307.

Hillis, A. E., Tuffiash, E., Wityk, R. J., \& Barker, P. B. (2002). Regions of neural dysfunction associated with impaired naming of actions and objects in acute stroke. Cognitive Neuropsychology, 19, 523-534.

Kable, J. W., Kimberg, D. Y., \& Chatterjee, A. (2004). Dealing with potential reaction time confounds in the interpretation of fMRI results. Paper presented at the Cognitive Neuroscience Society Eleventh Annual Meeting, San Francisco, CA.

Kable, J. W., Lease-Spellmeyer, J., \& Chatterjee, A. (2002). Neural substrates of action event knowledge. Journal of Cognitive Neuroscience, 14, 795-805.

Kan, I. P., Kable, J. W., van Scoyoc, A., Chatterjee, A., \& Thompson-Schill, S. L. (in press). Tools: Dissociable effects of motor experience and lexical competition. Journal of Cognitive Neuroscience.

Kellenbach, M. L., Brett, M., \& Patterson, K. (2003). Actions speak louder than functions: The importance of manipulability and action in tool representation. Journal of Cognitive Neuroscience, 15, 30-46.

Kraut, M. A., Moo, L. R., Segal, J. B., \& Hart, J., Jr. (2002). Neural activation during an explicit categorization task: Category- or feature-specific effects? Brain Research, Cognitive Brain Research, 13, 213-220.

Martin, A., Haxby, J. V., Lalonde, F. M., Wiggs, C. L., \& Ungerleider, L. G. (1995). Discrete cortical regions associated with knowledge of color and knowledge of action. Science, 270, 102-105.

McCarthy, R., \& Warrington, E. K. (1985). Category specificity in an agrammatic patient: The relative impairment of verb retrieval and comprehension. Neuropsychologia, 23, 709-727.

McDonald, C. R., Crosson, B., Valenstein, E., \& Bowers, D. (2001). Verbal encoding deficits in a patient with a left retrosplenial lesion. Neurocase, 7, 407-417.

McRae, K., de Sa, V. R., \& Seidenberg, M. S. (1997). On the nature and scope of featural representations of word meaning. Journal of Experimental Psychology: General, 126, 99-130. 
Mecklinger, A., Gruenewald, C., Besson, M., Magnie, M. N., \& Von Cramon, D. Y. (2002). Separable neuronal circuitries for manipulable and non-manipulable objects in working memory. Cerebral Cortex, 12, 1115-1123.

Miceli, G., Silveri, M. C., Nocentini, U., \& Caramazza, A. (1988). Patterns of dissociation in comprehension and production of nouns and verbs. Aphasiology, 2, 351-358.

Mitchell, J. P., Heatherton, T. F., \& Macrae, C. N. (2002). Distinct neural systems subserve person and object knowledge. Proceedings of the National Academy of Sciences, U.S.A., 99, 15238-15243.

Peigneux, P., Salmon, E., van der Linden, M., Garraux, G., Aerts, J., Delfiore, G., Degueldre, C., Luxen, A., Orban, G., \& Franck, G. (2000). The role of lateral occipitotemporal junction and area MT/V5 in the visual analysis of upper-limb postures. Neuroimage, 11, 644-655.

Perani, D., Cappa, S. F., Bettinardi, V., Bressi, S., GornoTempini, M., Matarrese, M., \& Fazio, F. (1995). Different neural systems for the recognition of animals and man-made tools. NeuroReport, 6, 1637-1641.

Perani, D., Cappa, S. F., Schnur, T., Tettamanti, M., Collina, S., Rosa, M. M., \& Fazio, F. (1999). The neural correlates of verb and noun processing. A PET study. Brain, 122, 2337-2344

Perani, D., Schnur, T., Tettamanti, M., Gorno-Tempini, M., Cappa, S. F., \& Fazio, F. (1999). Word and picture matching: A PET study of semantic category effects. Neuropsychologia, 37, 293-306

Postle, B. R., Zarahn, E., \& D'Esposito, M. (2000). Using event-related fMRI to assess delay-period activity during performance of spatial and nonspatial working memory tasks. Brain Research, Brain Research Protocols, 5, 57-66.

Pulvermuller, F. (1999). Words in the brain's language. Behavioral and Brain Sciences, 22, 253-336.

Rizzolatti, G., Fogassi, L., \& Gallese, V. (2001).

Neurophysiological mechanisms underlying the understanding and imitation of action. Nature Reviews. Neuroscience, 2, 661-670.

Saffran, E. M., Coslett, H. B., \& Keener, M. T. (2003). Differences in word associations to pictures and words. Neuropsychologia, 41, 1541-1546.

Saffran, E. M., \& Schwartz, M. F. (1994). Of cabbages and things: Semantic memory from a neuropsychological perspective-A tutorial review. In C. Umilta \& M. Moscovitch (Eds.), Attention and performance XV: Conscious and nonconscious information processing (pp. 507-536). Cambridge: MIT Press.

Shallice, T., Fletcher, P., Frith, C. D., Grasby, P., Frackowiak, R. S., \& Dolan, R. J. (1994). Brain regions associated with acquisition and retrieval of verbal episodic memory. Nature, 368, 633-635.

Shapiro, K., \& Caramazza, A. (2003). The representation of grammatical categories in the brain. Trends in Cognitive Sciences, 7, 201-206.

Simmons, W. K., \& Barsalou, L. W. (2003). The similarity-intopography principle: Reconciling theories of conceptual deficits. Cognitive Neuropsychology, 20, 451-486.

Thompson-Schill, S. L. (2003). Neuroimaging studies of semantic memory: Inferring "how" from "where". Neuropsychologia, 41, 280-292.

Thompson-Schill, S. L., Aguirre, G. K., D'Esposito, M., \& Farah, M. J. (1999). A neural basis for category and modality specificity of semantic knowledge. Neuropsychologia, 37, 671-676.
Thompson-Schill, S. L., D'Esposito, M., Aguirre, G. K., \& Farah, M. J. (1997). Role of left inferior prefrontal cortex in retrieval of semantic knowledge: A reevaluation. Proceedings of the National Academy of Sciences, U.S.A., 94, 14792-14797.

Thompson-Schill, S. L., D'Esposito, M., \& Kan, I. P. (1999). Effects of repetition and competition on activity in left prefrontal cortex during word generation. Neuron, 23, 513-522.

Thompson-Schill, S. L., Swick, D., Farah, M. J., D'Esposito, M., Kan, I. P., \& Knight, R. T. (1998). Verb generation in patients with focal frontal lesions: A neuropsychological test of neuroimaging findings. Proceedings of the National Academy of Sciences, U.S.A., 95, 15855-15860.

Tootell, R. B., Reppas, J. B., Kwong, K. K., Malach, R., Born, R. T., Brady, T. J., Rosen, B. R., \& Belliveau, J. W. (1995). Functional analysis of human MT and related visual cortical areas using magnetic resonance imaging. Journal of Neuroscience, 15, 3215-3230.

Tranel, D., Adolphs, R., Damasio, H., \& Damasio, A. R. (2001). A neural basis for the retrieval of words for actions. Cognitive Neuropsychology, 18, 655-674.

Tranel, D., Damasio, H., \& Damasio, A. R. (1997). A neural basis for the retrieval of conceptual knowledge. Neuropsychologia, 35, 1319-1327.

Tranel, D., Kemmerer, D., Adolphs, R., Damasio, H., \& Damasio, A. R. (2003). Neural correlates of conceptual knowledge for actions. Cognitive Neuropsychology, 20, 409-432.

Tranel, D., Martin, C., Damasio, H., Grabowski, T. J., \& Hichwa, R. (in press). Effects of noun-verb homonymy on the neural correlates of naming concrete entities and actions. Brain and Language.

Tyler, L. K., Bright, P., Fletcher, P., \& Stamatakis, E. A. (2004). Neural processing of nouns and verbs: The role of inflectional morphology. Neuropsychologia, 42, 512-523.

Tyler, L. K., \& Moss, H. E. (2001). Towards a distributed account of conceptual knowledge. Trends in Cognitive Sciences, 5, 244-252.

Tyler, L. K., Russell, R., Fadili, J., \& Moss, H. E. (2001). The neural representation of nouns and verbs: PET studies. Brain, 124, 1619-1634

Tyler, L. K., Stamatakis, E. A., Dick, E., Bright, P., Fletcher, P., \& Moss, H. (2003). Objects and their actions: Evidence for a neurally distributed semantic system. Neuroimage, 18, 542-557.

Valenstein, E., Bowers, D., Verfaellie, M., Heilman, K. M., Day, A., \& Watson, R. T. (1987). Retrosplenial amnesia. Brain, 110, 1631-1646.

Vinson, D. P., \& Vigliocco, G. (2002). A semantic analysis of grammatic class impairments: Semantic representations of object nouns, action nouns and action verbs. Journal of Neurolinguistics, 15, 317-351.

Warrington, E. K., \& Shallice, T. (1984). Category specific semantic impairments. Brain, 107, 829-854.

Watson, J. D., Myers, R., Frackowiak, R. S., Hajnal, J. V., Woods, R. P., Mazziotta, J. C., Shipp, S., \& Zeki, S. (1993). Area V5 of the human brain: Evidence from a combined study using positron emission tomography and magnetic resonance imaging. Cerebral Cortex, 3, 79-94.

Zarahn, E., Aguirre, G. K., \& D'Esposito, M. (1997). Empirical analyses of BOLD fMRI statistics: I. Spatially unsmoothed data collected under null-hypothesis conditions. Neuroimage, 5, 179-197. 